\title{
Friendly Patrolling: A Model of Natural Encounters
}

\author{
Kotaro Hayashi $^{*+*}$, Masahiro Shiomi* ${ }^{*}$, Takayuki Kanda ${ }^{* *}$ and Norihiro Hagita ${ }^{* \dagger}$ \\ *ATR Intelligent Robotics and $\quad$ The Department of Information $\quad$ Japan Science and Technology \\ Communication Labs. \\ Science, NAIST \\ Agency, CREST
}

Email: \{hayashik, m-shiomi, kanda, hagita\}@atr.jp

\begin{abstract}
This study addresses encounter interactions in public environments where people and robots walk around. In daily life, security guards, police officers, and sales clerks roam around environments and nonverbally present friendly behavior so that people feel comfortable talking to them. We modeled the behavior of human experts during friendly patrolling, which we defined as a roaming behavior that nonverbally presents a friendly attitude, to encourage people to talk to such professionals. The model was implemented in a humanoid robot, Robovie, and tested in a shopping mall. The experimental results with 39 participants demonstrated that the model worked as intended.
\end{abstract}

\section{Keywords: component, human-robot interaction, social robots}

\section{INTRODUCTION}

Many robots have been developed in research projects for working in daily public environments. In a shopping center, a robot working as a shopping assistant approaches a customer to offer assistance [1]. On the street, a robot may talk to visitors and give directions [2]. Other robots work in different environments such as museums [3, 4] and hospitals [5].

How do these robots encounter people? Often, either a robot or a person walks up and talks to the other. For instance, when the robot's role is receptionist or information provider, the robot is naturally designed to wait at a fixed location. People approach the robot and initiate interaction $[2,6]$. On the other hand, researchers have also designed robots that initiate interaction [7] when they are used as helpers or advertising staff. Yamazaki et al. modeled the behavior of helpers in homes for the elderly and developed a robot that exhibits its availability to help [8]. Kanda et al. developed a method in which a robot approaches people who are walking by anticipating their trajectories [9].

However, in such a public environment as a shopping mall (Fig. 1), encounters are more dynamic and complex, including many encounters while people are walking. For example, when a person wants to know a shop's location, he might approach the shop staff to ask if the shop staff is not busy and seems friendly. Such interaction is seemingly motivated by the person who wants to know the location, but it is also largely affected by the nonverbal behavior exhibited by the shop staff. The mall visitor might not approach the shop staff unless he thinks the shop staff is friendly and receptive. It is essential that mobile robots show their availability in friendly ways. Many potential applications for robots require this capability, for instance, shopkeepers in supermarkets, airport helpers, and guards on streets and at public facilities.
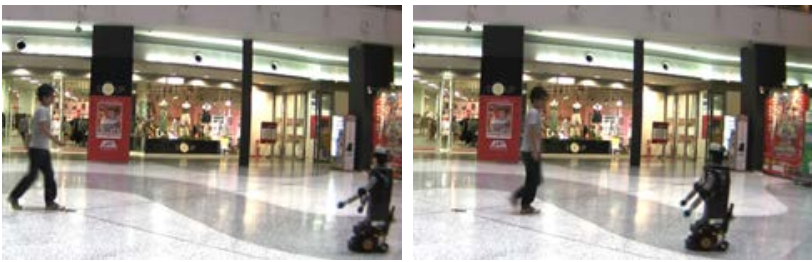

Figure 1. Encounter between robot and a person walking

We name such interaction friendly patrolling, i.e., nonverbally presenting a friendly attitude while roaming around an environment for surveillance, assistance, or security. Friendly patrolling is an expected behavior for natural encounters with people in such services as clerks in shops, staff at train and bus stations, and guards and police officers in public environments in some countries (hopefully in many countries). On the other hands, greeting all people [7] is too aggressive to be friendly patrolling. We might feel uncomfortable and obstructed if a guard were to approach us to offer assistance when we are simply walking through an environment.

We modeled the friendly patrolling behaviors of people with experience as guards. They did not initiate conversations with visitors, but they did not ignore them, either. They nonverbally showed their availability and friendliness by changing speed, gaze, and the trajectory shape when someone is present.. They took a path to approach visitors and oriented their gaze to them, but only from a distance outside the social/conversational sphere. The modeled behavior was implemented in a humanoid robot and tested in an experiment.

\section{RELATED WORKS}

\section{A. Human communication}

In the area of human communication, few studies have addressed the initiation of interaction. Humans start interaction with greetings. Goffman reported that eye-contact is one of the most important social rules for accepting or refusing the approaches of others [10]. Kendon reported that when people initiate interaction, they exchange greetings two times; the first greetings are conducted nonverbally at a distance, and the second verbal greetings are conducted at a closer distance with a smile [11].

Our study focused on realizing natural encounters for a robot in a situation where both robots and people are walking in a public environment. Based on Kendon's work, we use eyecontact to model a method for realizing friendly patrolling. In addition, we analyzed how people walk in public environments to show that they are available to help others.

This research was sponsored by JST, CREST. 


\section{B. Navigation techniques in presence of humans}

Proxemics, which is one focus in HRI, includes studies about personal space [12], approach methods [13], and spatial formation [14]. Based on findings from HRI as well as human science, researchers have developed navigation techniques in the presence of humans. Sisbot et al. developed a path planning technique that considers the human preference for the location of robots [15]. Their robot refrains from standing close behind a person or suddenly leaping from a concealed place to avoid startling people in the environment. Pacchierotti et al. developed a path planning technique to pass through a hallway without obstructing people's walking path [16]. In a simulation, Henry et al. used a learning technique to adapt the robot's trajectory to the flow of walking people [17]. Studies have already addressed the navigation techniques for avoiding [18], guiding [19], and following people [20].

Our study modeled human navigation in a friendly patrolling context. None of the previous studies addressed a method to perform friendly patrolling. We believe that our study overlaps with these previous studies and could be integrated in the future, e.g., to plan friendly patrolling with our technique and to avoid obstructing other pedestrians with the existing techniques.

\section{Model of encounter}

Two techniques facilitate encounters between robots and people. Michalowski et al. analyzed people's standing positions around a receptionist robot and categorized them in relation to people's participation roles in the interaction [6]. Bergström et al. designed a robot's reaction to the behavior of people approaching it to facilitate the initiation of interaction [21]. They modeled a robot-initiating type of encounter and built a technique to predict people's trajectories so that a robot approached from the frontal direction to bring itself to people's attention [9, 7].

The concept of displaying availability was well presented by Yamazaki et al. [8], who precisely analyzed the initiation of interaction between caregivers and senior citizens in homes for the elderly. They found that caregivers exhibit availability by directing their gaze toward someone who might need help. We found a pattern of showing availability by directing gaze common to friendly patrolling. Their study addressed the context in a home for the elderly where they sit and wait for service from caregivers; however, their study did not address situations where people walk around the environment.

\section{MODELING FRIENDLY PATROLLING}

In some countries, such as Japan, guards often engage in friendly patrolling since their expected role is to patrol for security reasons and provide guide information. Hence, we decided to model friendly patrolling on our observations of the walking behavior of people who have experience as shopping mall guards.

\section{A. Data collection}

1) Procedure: We employed two people with experience as security guards (hereinafter guard). Data were collected in the daytime in a $470 \mathrm{~m}^{2}$ open space in a shopping mall (Fig. 2) where a few pedestrians pass by every minute.

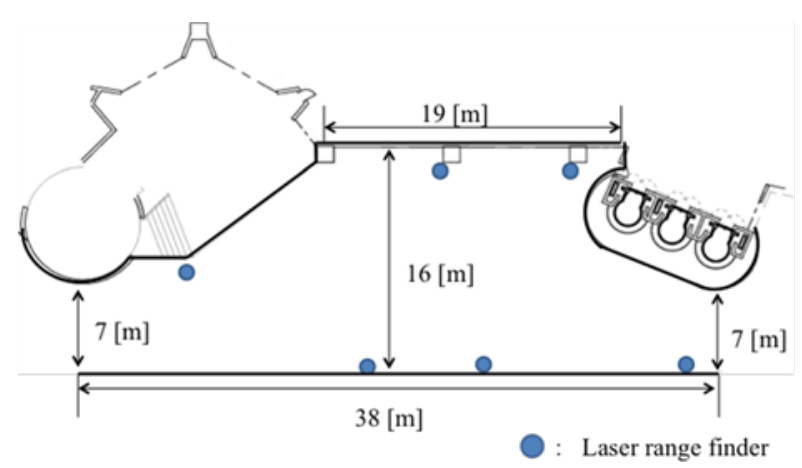

Figure 2. Map of open space in shopping mall

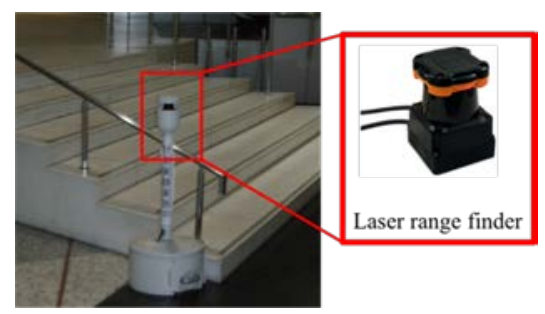

Figure 3. Position estimation system

To investigate the features of friendly patrolling, we prepared two different kinds of instructions: busy patrolling and friendly patrolling. Each guard individually walked around the environment during a 10-minute period following each instruction in this order:

- Friendly patrolling: Each guard walked around the environment to allow people to communicate easily.

- Busy patrolling: Each guard walked around the environment in a busy manner, showing friendliness but not showing availability thus would provide impression that it is not easy-to-talk. Busy patrolling was prepared as a counterpart to friendly patrolling, instead of unfriendly patrolling. Pragmatically, guards have two busy situations (e.g., going to a particular location to respond to a problem) or non-busy. During non-busy situations, they are supposed to be friendly.

2) Recording data: Position data and video of both the guards and pedestrians were collected. The position data were used to analyze how the guards walked around and encountered pedestrians.

a) Position estimation system: For this purpose, we used Hokuyo UTM-30LX laser range finders mounted at $85 \mathrm{~cm}$ around the trial area's perimeter to track people's positions (Fig. 3). This tracking technique provides highly stable and reliable trajectory data. For natural walking speeds, the tracking accuracy of our sensor configuration was measured to be $+/-6 \mathrm{~cm}$ [22].

b) Video recording: Videos were recorded to analyze looking behavior, which is a well known trigger for indicating the timing and initiating conversations in the research field of HRI [8, 23, 24, 25]. 


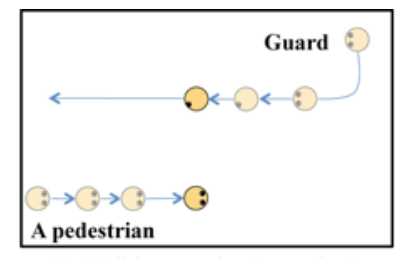

(a) Walking nearby (up to $3 \mathrm{~m}$ )

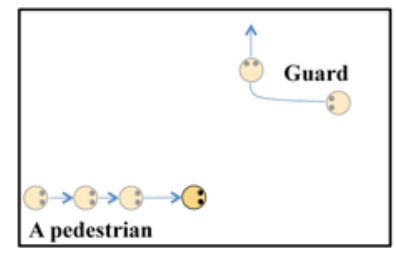

(c) U-turn

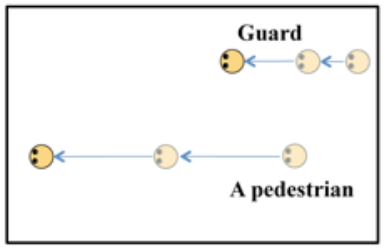

(e) Approached, but failed to catch up

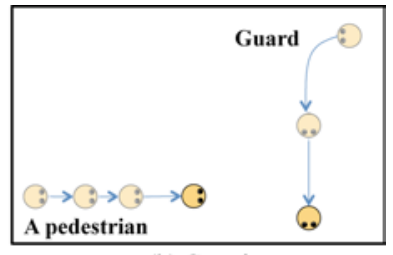

(b) Crossing

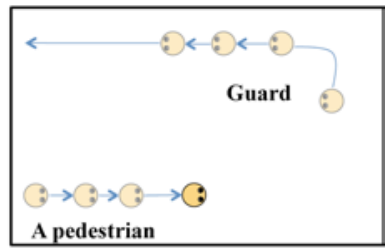

(d) Passing (from $3 \mathrm{~m}$ on)

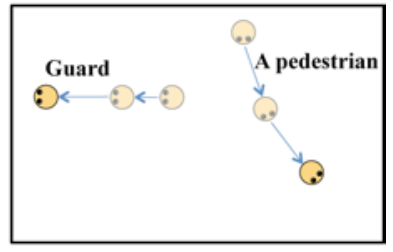

(f) Did not notice
Figure 4. Walking patterns

\section{B. Analysis of friendly patrolling}

1) Comparison of busy and friendly patrolling: First, we observed the walking behaviors of the guards between busy and friendly patrolling. We analyzed the features of each patrolling situation from four points of view: speed, gaze, the trajectory shape when there is no one nearby, and its shape when someone is present. To analyze the data, we used the estimated position data and recorded videos.

As a result, there are three differences between friendly and busy patrolling: speed, gaze, and the trajectory shape when someone is present. Note that the shape of the trajectory when there is no one nearby in the environment was not different between the settings; the guards went around the environment. Therefore, below we describe a detailed comparison of the speed and the trajectory when someone is present.

a) Walking speed when nobody is present: The average walking speeds of the guards were calculated using the estimated position data. The calculation results indicate that the guards used the same trajectory shape but a different speed in each patrolling situation when there was no one nearby in the environment: $1.3 \mathrm{~m} / \mathrm{sec}$ for busy and $0.8 \mathrm{~m} / \mathrm{sec}$ for friendly patrolling.

b) Changes in walking trajectory: When someone entered the environment, the guards changed their walking trajectories; however, the changes in trajectories between friendly and busy patrolling were different.

After data collection, we conducted a preliminary analysis and found that their behavior largely differs based on situations whether there was a pedestrian, and specifically whether the pedestrian was visible to and within a certain distance from a guard (thus, if he wanted he was able to come close to the pedestrian). To analyze the detailed differences of the
TABLE I. FEATURES BETWEEN FRIENDLY AND BUSY PATROLLING

\begin{tabular}{|c|l|c|c|}
\hline & & Friendly & Busy \\
\hline \hline \multirow{4}{*}{$\begin{array}{c}\text { (A) Guard could } \\
\text { come close if he } \\
\text { wanted }\end{array}$} & (a) Walking nearby (up to 3 m) & $9 / 10$ & $4 / 13$ \\
\cline { 2 - 4 } & (b) Crossing & $0 / 10$ & $2 / 13$ \\
\cline { 2 - 4 } & (c) U-turn & $0 / 10$ & $2 / 13$ \\
\cline { 2 - 4 } & (d) Passing (from 3 m on) & $1 / 10$ & $5 / 13$ \\
\hline \hline $\begin{array}{c}\text { (B) Guard } \\
\text { couldn't come } \\
\text { close even if he } \\
\text { wanted }\end{array}$ & $\begin{array}{l}\text { (e) Moved toward pedestrian, } \\
\text { but did not come enough close }\end{array}$ & $8 / 18$ & $2 / 9$ \\
\cline { 2 - 4 } & (f) Did not notice & $10 / 18$ & $7 / 9$ \\
\hline
\end{tabular}

trajectory changes, two coders classified the walking situations where someone entered the environment into two cases: (A) where a pedestrian was visible to a guard and within a distance where he could come close to the pedestrian if he wanted (possible to come close situation), and (B) the opposite case where the pedestrian was not visible to a guard (because the guard was facing another direction than the pedestrian's position) or too far from the pedestrian. Our interest in modeling friendly patrolling is in the former case; the classification results indicate ten friendly patrolling cases and 13 busy patrolling cases where it was possible for a guard to come close to a pedestrian.

The coders further classified them into subcategories. As shown in Fig. 4, they defined four categories for a case where a guard was able to come close ${ }^{1}$ :

- Walking nearby (up to $3 \mathrm{~m}$ ): Guards were walking nearby a pedestrian and the distance for encountering was less than $3 \mathrm{~m}$.

- Crossing: Guards crossed in front of a pedestrian.

- U-turn: Guards turned to go away from a pedestrian.

- Passing (from $3 \mathrm{~m}$ on): Guards did not walk nearby a pedestrian, and the distance for encountering was more than $3 \mathrm{~m}$.

Moreover, they defined two categories for a case where a guard was not able to come close:

- Moved toward the pedestrian, but did not come enough close: Guards moved toward the pedestrian but they could not come enough close to the pedestrian because the pedestrian walked faster than the guard or the pedestrian was too far from the guards.

- Did not notice: Guards did not notice the pedestrians' arrival.

Cohen's kappa coefficient from the two coders' classifications was 0.934 , indicating that their evaluations was highly consistent. After classification, to analyze consistent trajectories for modeling, the two coders discussed and reached

\footnotetext{
${ }^{1}$ The number of encounters differed between conditions because there was some divergence in the number of visitors in each 10-minute recording; thus, we need to look at the difference in ratio rather than number.
} 
a consensus in their classification results for the entire coding process.

The coding results show that the guards often walked nearby a pedestrian in friendly patrolling $(9 / 10)$ in case (A), which is where the guards were able to come close to the pedestrian. On the other hand, they did not walk nearby a pedestrian so frequently during busy patrolling (4/13). There was little difference in cases (B). Therefore, these results indicate that walking trajectories between friendly and busy patrolling have differences when a guard was able to come close.

In summary, we found three kinds of differences between friendly patrolling and busy patrolling: speed, gaze, and the trajectory shape when someone is present in the environment. These are the only factors in which we found differences; we did not find differences in other factors such as the shape of the trajectory when there is no one nearby. In friendly patrolling, guards often walked nearby a pedestrian at a slower speed than busy patrolling. Therefore, we decided to use this type of encounter behavior as our model for friendly patrolling (Fig. 4(a)).

2) Retrieving parameters: After comparing the guards' walking behavior between the two settings, we decided to focus on an encounter behavior to model friendly patrolling in which the guards encounter pedestrians from the front (Fig. 4(a)). We measured three factors (path separation distance, walking speed, and looking) for a 5-second time period before the timing of an encounter (Fig. 5), because they did not change these factors until a 5-second time period before. The horizontal distance means the distance between the robot and the pedestrian on the $\mathrm{x}$-axis of Fig. 5.

Path separation distance, which is the distance perpendicular to the direction of the movement, was calculated by using position data from the human tracking system. During encounters, the guards maintained a constant distance from the pedestrians; the average distance was $2.6 \mathrm{~m}$.

We also measured the average speed per second when the guards were encountering pedestrians from the front. As a result, the guards decreased their average speed from 650 to $570 \mathrm{~mm} / \mathrm{sec}$ one second before the timing of an encounter (Fig. 6). This indicates that the guards immediately decreased their speed when the horizontal distance to the pedestrian became less than about $3 \mathrm{~m}$.

Looking behavior during friendly patrolling was also analyzed using recorded video from the data collection. The guards started to look at the pedestrians when the horizontal distance between them became less than about $10 \mathrm{~m}$. Moreover, the guards limited their face direction; the maximum degree was around $45^{\circ}$ from the walking direction.

From these analyses, we defined a model for friendly patrolling as follows: (1) changing the walking direction to encounter the pedestrian from the front by taking a constant path separation distance, (2) decreasing walking speed, (3) looking at the pedestrian when the horizontal distance is less than $10 \mathrm{~m}$ with a maximum face direction of $45^{\circ}$, and (4) decreasing speed again when the horizontal distance became less than $3 \mathrm{~m}$.

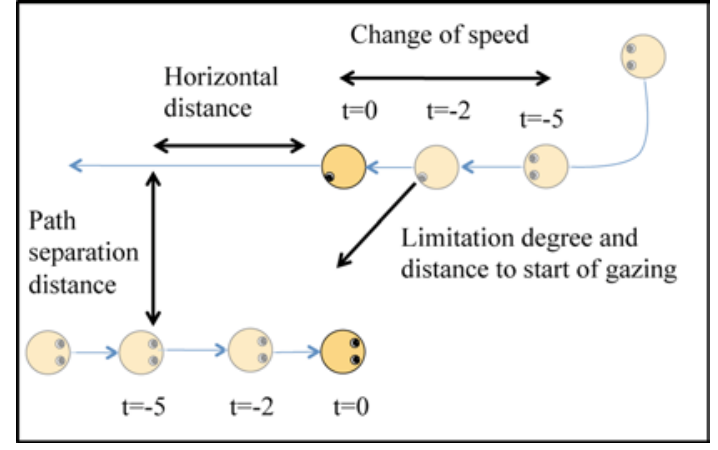

Figure 5. Analyzed parameters for modeling

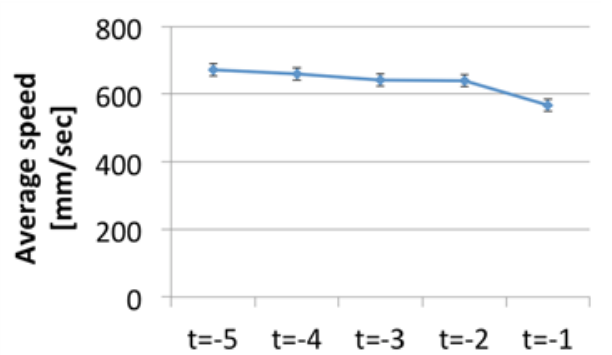

Figure 6. Average speed for a 5-second time period before timing of encounter

\section{Implementation of friendly patrolling}

We implemented this model on a mobile robot. We used a mobile humanoid and the human tracking system [22] that was used for data collection described in Section 3.1.2. The system was also used for robot localization to estimate the robot's position as accurately as the people's positions.

1) Robovie: To implement the model, we used Robovie, an interactive humanoid robot characterized by its humanlike physical expressions and its various sensors. Robovie is 120 $\mathrm{cm}$ high and $40 \mathrm{~cm}$ wide.

Its mobile base is a Pioneer 3-DX (Active Media). Its maximum moving speed was set to $800 \mathrm{~mm} / \mathrm{second}$, based on the average speed of people walking in a mall, the mobile base's capability, and safety concerns. For obstacle detection, we attached a Hokuyo URG-04LX laser range finder to the lower part of its mobile base to detect low obstacles that the environmental sensors cannot detect. For example, if a child runs too close to the front of the robot, the attached LRF can immediately stop the robot.

2) Friendly patrolling controller: This controller has two functions to realize friendly patrolling: a locomotion controller and a gaze controller.

a) Locomotion controller: it plans the robot's path and controls its speed for encountering a target pedestrian from the front.

To provide the path, the controller predicts the future moving trajectory of the target by a simple linear prediction. It uses the positions from the past two seconds and assumes the pedestrian will move straight; i.e., it draws a line with a linear interpolation and assumes this line is the future path of the 


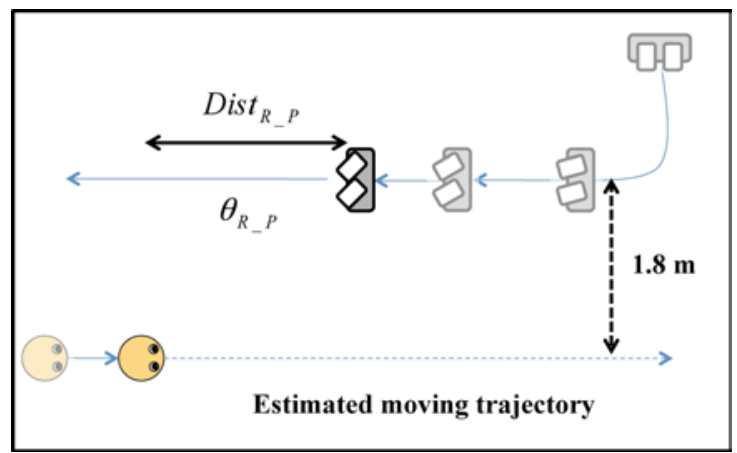

Figure 7. Locomotion path and parameters for friendly patrolling

pedestrian. This is simplistic assumption for general situations, although we found it reasonably works in the corridor situations we conducted the experiment, since in a long corridor people usually walk on a straight line.

Path planning was conducted, as illustrated in Fig. 7. In the beginning, the robot moves vertically to the parallel path's line, which is parallel with the predicted path of the pedestrian with the path separation distance. Then it briefly follows an asymptote to orient toward the pedestrian and moves along the parallel path.

Its speed is controlled with the following equation:

$$
\text { Speed }= \begin{cases}S_{f_{-} \text {far }} & \left(\text { Dist }_{R_{-} P}>\text { Dist }_{f}\right) \\ S_{f_{-} \text {near }} & \left(\text { Dist }_{R_{-} P}<\text { Dist }_{f}\right),\end{cases}
$$

where $S_{f f a r}$ is the speed for friendly patrolling, $S_{f \text { near }}$ is the speed when the robot is near the pedestrian, $\operatorname{Dist}_{R_{P} P}$ is the horizontal distance between the robot and the target pedestrian shown in Fig. 7, and Dist is $_{f}$ the distance threshold to change the speed. When nobody is present in the environment, the robot speed is set to idle, which is the same speed as the friendly patrolling of the guards: $800 \mathrm{~mm} / \mathrm{sec}$. We designed the robot to stop for safety when the distance between itself and the nearest object is less than $500 \mathrm{~mm}$.

b) Gaze controller: it controls the robot's face directions, FD $\left(0^{\circ}\right.$ indicates the front of the robot). It follows the model built in Section 3.2, limits the gaze angle to $45^{\circ}$, and only looks at the person within a certain range of distance, described in the following equation:

$$
F D=\left\{\begin{array}{lr}
0 & \left(\text { Dist }_{R_{-} P}>\text { Dist }_{g}\right) \\
\theta_{R_{-} P} & \left(\text { Dist }_{R_{-} P}<\text { Dist }_{g} \& \&-45<\theta_{R_{-} P}<45\right) \\
45 & \left(\text { Dist }_{R_{-} P}<\text { Dist }_{g} \& \& \theta_{R_{-} P}>45\right) \\
-45 & \left(\text { Dist }_{R_{-} P}<\text { Dist }_{g} \& \& \theta_{R_{-} P}<-45\right)
\end{array},\right.
$$

where Dist $_{g}$ is the distance threshold to start facing and $\theta_{R_{-} P}$ is the angle between the robot and the target (Fig. 7). When there is no one nearby in the environment, the face direction of the robot is fixed to the front of it.

We conducted a pre-trial to adjust the parameters to implement the model into the robot. In the pre-trial, we changed each parameter then asked impressions to participants who are recruited as the same way of our experiment as written in section IV.B. Finally, we used a $S_{f f a r}$ of $650 \mathrm{~mm} / \mathrm{sec}$, which equaled the human guards'. Since in pre-trials participants did not perceive the robot's $570 \mathrm{~mm} / \mathrm{sec} S_{f \text { near }}$ as clearly slower than $S_{f f a r}$, we tested a slower $S_{f \text { near }}$ until participants perceived a speed decrease. Finally, $500 \mathrm{~mm} / \mathrm{sec}$ was chosen for $S_{f \text { near }}$. We found that we needed to adjust the distance parameters too, otherwise participants felt that the robot was too far from them to talk. This seems to reflect the shortness of the robot's height. With pre-trials, we also tested a range of distance parameters and chose those that provided the best impression on friendly patrolling, i.e., less obtrusive and easy-to-talk. We finally chose a path separation distance to $1.8 \mathrm{~m}$, Dist $_{f}$ to $2 \mathrm{~m}$, and Dist $_{g}$ to $7 \mathrm{~m}$.

\section{EXPERIMENT}

We experimentally measured the effect of the friendly patrolling model in a robot that encounters people in a shopping mall.

\section{A. Experiment Design and Hypothesis}

Our research focus is the easy-to-talk behavior during roaming that is pragmatically used in applications that combine the roaming task (e.g., for surveillance and security) and the information-providing task. In daily life we can see such human examples as staff that roam around supermarkets and guards who patrol airports. When such applications are replicated in a robot, we must prepare it to navigate on a predefined path or on one that is generated dynamically but independent from the location of people (e.g., a path generation algorithm for efficiency in swarm robots [26]).

Therefore, we compared our model with patrolling on a pre-defined path instead of busy-patrolling. Here, depending on the walking direction of the people, we prepared two conditions: round trip and crossing (Section 4.3). We prepared two conditions for a pre-defined path because the two conditions correspond to two representing directions people meet with the robot. Particularly, the round trip would be likely to happen if both a robot and a visitor roam along a corridor; the robot comes to them on a parallel trajectory from a distance.

Our hypothesis is that our model will outperform such a patrolling method with a pre-defined path. In addition, we hypothesized that the gaze behavior will help the robot provide an easy-to-talk impression, which was found to be useful in other situations [8, 23, 24, 25].

\section{B. Participants}

Participants included 39 Japanese people (20 men and 19 women whose average age was 28.5 years, S.D. was 11.2 ) who had not previously interacted with our robots. They were paid 3,000 yen (approx. 36 USD) for two hours of participation.

\section{Conditions}

We conducted a $2 \times 3$ within-participants factorial design with two factors: gaze and path. Both factors were designed to compare the difference of contingency, which was expected to be an important factor for friendly patrolling. 


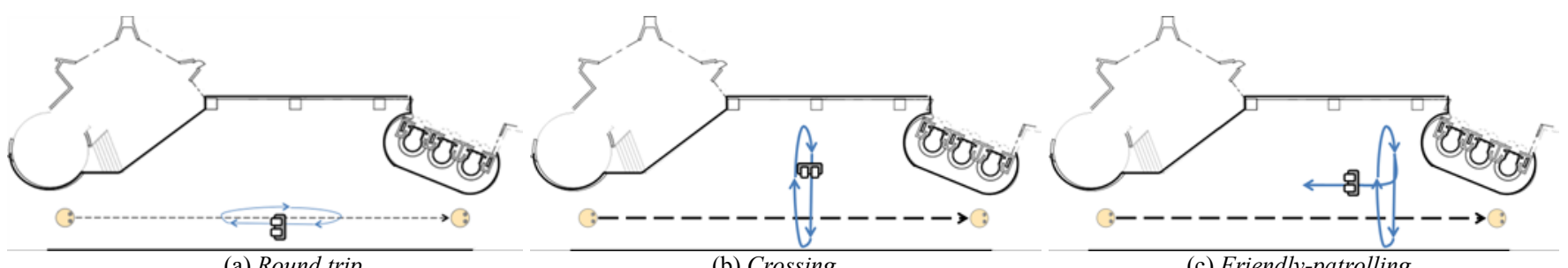

(a) Round trip

(b) Crossing

(c) Friendly-patrolling

Figure 8. Movements in each condition

\section{1) Gaze factor:}

a) With-gaze condition: The robot looked at the participant, as described in Section 3.3.2.

b) Without-gaze condition: The robot did not look at the participant. The face direction of the robot was fixed on the front.

\section{2) Path factor:}

a) Round trip condition: The robot turned on a straight line that was defined beforehand as the main corridor of the environment. The robot's speed was fixed to $800 \mathrm{~mm} / \mathrm{sec}$. An example of a moving path is shown in Fig. 8(a).

b) Crossing condition: The robot crossed at right angles to the environment's main corridor. Its speed was also fixed to $800 \mathrm{~mm} / \mathrm{sec}$. An example moving-path is shown in Fig. 8(b).

c) Friendly-patrolling condition: The robot encountered the participant from the front based on the calculated path described in Section 3.3.2. Before the participants entered the environment, the robot's movement resembled the crossing condition; the robot crossed at right angles to the main corridor of the environment at $800 \mathrm{~mm} / \mathrm{sec}$. After the participant entered the environment, the robot changed its moving trajectory and speed. In other words, the robot only showed contingency in its walking in this condition. An example of a moving path is shown in Fig. 8(c). A video on this condition is available on http://www.youtube.com/watch?v=OI82JQgJvNA

\section{Procedure}

The experiment was conducted in the same shopping mall at which we gathered the walking data for modeling. We also installed the position estimation system described in Section 3.1.2 to the environment. Each session was scheduled in the daytime on weekdays to avoid extra crowds that might be caused by the robot.

Since this has a within-participant design, each participant engaged in six sessions. Before the first session, they were given a brief description of the experiment's purpose and procedure. We explained the start and end points and the main corridor to walk through the environment and conducted a practice walk from the start to the end points.

Moreover, we told the participants to stay at the start point for a few seconds before each session. While they were waiting, the robot was roaming around based on the condition. After a few seconds of waiting, the participants were able to enter and walk through the environment at any time.

A staff member set the participant as an encounter target for the robot during this waiting time; after deciding the target, the robot was completely autonomous to decide its moving trajectory, speed and face direction.

After each session, they filled out questionnaires in which the participants considered such various walking situations as busy walking or window-shopping when they evaluated the robot.

The order of these conditions was counterbalanced. For safety, the staff remained around the robot during the trials.

\section{E. Measurement}

Friendly patrolling is a behavior that we would expect for a natural encounter; i.e., a robot tries to make pedestrians feel comfortable when they are unobtrusively talking to it. Therefore, to evaluate the availability of the robot and whether the encounter with the robot is obstructive to the participants in each condition, we prepared three kinds of questionnaires to measure the subjective impressions of the participants: obstruction-free degree of the robot (whether people did not feel hindered in the robot's movements or not), ease of talking to the robot, and total evaluation of the robot. All questionnaire items were on a 1-to-7 point scale where 7 presents the most positive, 4 is neutral, and 1 is the most negative.

\section{F. Predictions}

The observations of the guard's friendly patrolling behavior lead to a hypothesis that the friendly patrolling path would increase the obstruction-free degree of the robot and the ease of speaking to it. Regarding gaze, as previous literature indicates [8], a robot's looking behavior would increase the ease of speaking to it. Thus, overall, a robot with both friendly patrolling and with-gaze is the most preferred in the total evaluation of the robot. Based on the above consideration, we made the following predictions:

- Prediction 1: Friendly patrolling will be rated high in obstruction-free degree of the robot and ease of speaking to the robot.

- Prediction 2: A robot equipped with-gaze will be rated high in ease of speaking to the robot.

- Prediction 3: A robot with both friendly patrolling and with-gaze will be rated high in total evaluation.

\section{RESULTS}

Figure 9 shows the results of the obstruction-free degree of the robot, the ease of speaking to it, and the total impressions of it. First, to test the prediction, we conducted a two-way repeated-measures ANOVA with two within-subject factors for obstruction-free degree of the robot, ease of speaking, and the 
(a) Obstruction-free degree

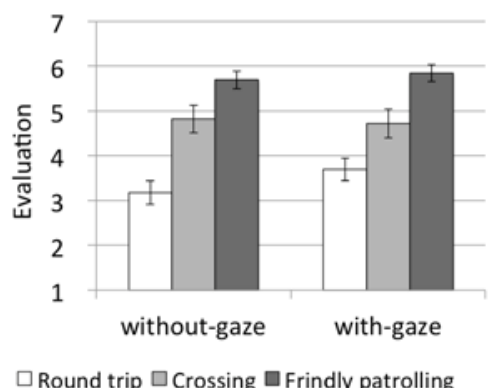

(b) Easy of talking

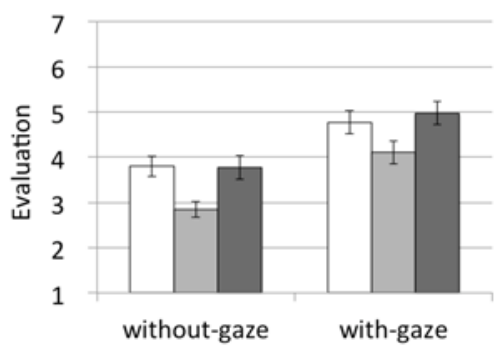

Figure 9. Questionnaire results
(C) Total evaluation

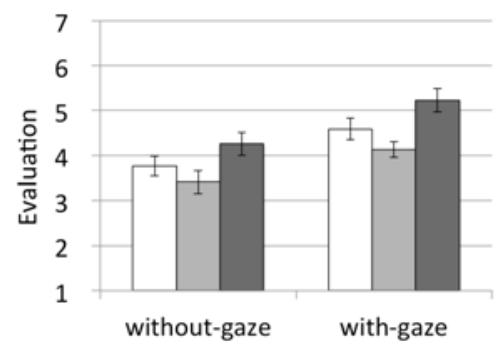

total impressions: gaze and path. Note that as far as we asked no one reported intentional bias toward the proposed condition.

Concerning the obstruction-free degree of the robot, a significant main effect was revealed in the path factor $(F(2$, 76) $=43.628, p<.001$, partial $\eta^{2}=.702$ ). Multiple comparisons with the Bonferroni method revealed significant differences: friendly patrolling $>$ round trips $(p<.001)$, friendly patrolling $>$ crossing $(p=.001)$, and crossing $>$ round trips $(p<.001)$. No significance was found in the gaze factor $(F(1,38)=1.013$, $p=.321$, partial $\left.\eta^{2}=.026\right)$ and in the interaction within these factors $\left(F(2,76)=.535, p=.590\right.$, partial $\left.\eta^{2}=.028\right)$. This means that friendly patrolling significantly increased the obstructionfree degree of the robot.

Concerning the ease of talking to the robot, a significant main effect was revealed in the gaze factor $(F(1,38)=31.604$, $p<.001$, partial $\left.\eta^{2}=.454\right)$. A significant main effect was also revealed in the path type $(F(2,76)=16.567, p<.001$, partial $\eta^{2}=.472$ ). Multiple comparisons with the Bonferroni method revealed significant differences: friendly patrolling $>$ crossing $(p<.001)$, round trips $>$ crossing $(p=.001)$, but no significant differences between friendly patrolling and round trips $(p=1.00)$. No significance was found in the interaction within these factors $\left(F(2,76)=0.273, \mathrm{p}=.762\right.$, partial $\left.\eta^{2}=.015\right)$. This means that friendly patrolling and round trip significantly increased the ease of talking to the robot. The gaze factor also significantly increased the ease of talking to the robot. Thus, these results support predictions 1 and 2 .

Concerning the total evaluation of the robot, a significant main effect was revealed in the gaze type $(F(1,38)=21.980$, $p<.001$, partial $\left.\eta^{2}=.366\right)$. A significant main effect was also revealed in the path type $(F(2,76)=12.825, p<.001$, partial $\eta^{2}=.409$ ). Multiple comparisons with the Bonferroni method revealed significant differences: friendly patrolling $>$ round trip $(p=.042)$, friendly patrolling $>$ crossing $(p<.001)$, but no significant differences between round trip and crossing $(p=.180)$. No significance was found in the interaction within these factors $\left(F(2,76)=0.238, p=.789\right.$, partial $\left.\eta^{2}=.013\right)$. This means that friendly patrolling significantly increased the total evaluation of the robot. Moreover, the gaze factor also significantly increased the total evaluation of the robot. Thus, these results support prediction 3 .

\section{DISCUSSION}

\section{A. How to use "friendly patrolling" in a real situation?}

This study addresses the interaction between a pedestrian and a robot to stay within a proof-of-concept environment to investigate the effects of friendly patrolling. In contrast, in the real world, situations rarely have only a single pedestrian in a large corridor. Will the friendly patrolling framework be applicable to a real environment?

In a corridor, where people's trajectories are rather predictable, this would be easily applied. Even though roaming on the main path would be obtrusive, as the experimental results showed, a robot could compute a path where it could encounter many people in the way that we modeled friendly roaming, which would probably be a path to the side of the main path where numerous people are walking. In a narrow corridor, the distance parameter might need to be adjusted; yet we believe that the basic concept remains applicable.

In a hall or a square, where people's motions are more complicated, this problem becomes more difficult. If the environment is not crowded, it could still be applied, although it would probably require a good global-planning of the trajectory based on a good guess of people's arrivals.

If the environment were more crowded, we would probably need further research to see whether our friendly patrolling remains friendly to the people surrounding it but who did not directly interact with it.

\section{B. Where will friendly patrolling be used?}

In this study, modeling was conducted with guards because the robots will be used for surveillance and security in such facilities as airports, train stations, malls, and on streets. But, will these robots simply patrol and intimidate people? We believe that such robots in the future will be used in friendly and socially acceptable manners. In other words, these robots need a function to show their friendliness. We believe that friendly patrolling will be a basic capability for patrolling robots.

Moreover, in the future when robots are used in such commercial facilities as shopping mall and supermarkets, they might have roles beyond surveillance and security, like human shop staff. For example, a shopkeeper robot might be reallocated from one area to another, or a delivery robot might move toward a shop for a delivery. Even for such robots, 
friendly patrolling will be useful. The robot's merits are multipurpose. If a robot has the capability to provide information, it spares time for people to provide information, as human workers do; or these robots might need to show a busy-state with a busy patrolling method, which was not modeled in the study, but this study provides a insight for the counterpart of friendly patrolling, too.

\section{Limitations}

Since the findings in this study are limited to a single pedestrian situation, the effect of friendly patrolling on a person who does not directly interact with the robot was not tested and requires further testing in the future. We believe that this will clarify when the idea might be used in a real field environment.

Two parameters were prepared in the study: the speed when the robot is near a pedestrian and the path separation distance. These parameters are probably dependent on the robot we used and perhaps Japanese people. When it is used with other settings, the parameters should be adjusted. For example, a taller robot might need a longer distance between paths.

\section{CONCLUSION}

This paper addresses a friendly patrolling model that enables a robot to naturally encounter people in real environments, such as shopping malls. For this purpose, we modeled the walking behavior of people who have actual experience working as guards in Japan. We analyzed the behavior related to this position information and gaze behavior and implemented a friendly patrolling model for a humanoid robot. Our experiment in a real shopping mall revealed that both gaze and friendly patrolling contributed to achieving natural encounters with people.

As a supplementary material, a scene of friendly patrolling in a corridor of a shopping mall is available here:

http://www.youtube.com/watch?v=OI82JQgJvNA

\section{REFERENCES}

[1] Gross, H.M., Boehme, H. J., Schroeter, C., Mueller, S., Koenig, A., Martin, C., Merten, M., and Bley, A. Shopbot: Progress in Developing an Interactive Mobile Shopping Assistant for Everyday Use, IEEE Int. Conf. on Systems, Man, and Cybernetics, pp. 3471-3478, 2008.

[2] Kanda, T., Shiomi, M., Miyashita, Z., Ishiguro H., and Hagita, N. An Affective Guide Robot in a Shopping Mall, ACM/IEEE Int. Conf. on Human-Robot Interaction, pp. 173-180, 2009.

[3] Burgard, W., Cremers, A. B., Fox, D., Hahnel, D., Lakemeyer, G., Schulz, D., Steiner, W., and Thrun, S. The Interactive Museum TourGuide Robot, National Conf. on Artificial Intelligence, pp. 11-18, 1998.

[4] Thrun, S., Bennewitz, M., Burgard, W., Cremers, A. B., Dellaert, F., Fox, D., Hähnel, D., Rosenberg, C., Roy, N., Schulte, J., and Schulz, D. Minerva: A Second-Generation Museum Tour-Guide Robot, IEEE Int. Conf. on Robotics and Automation, pp. 1999-2005, 1999.

[5] Montemerlo, M., Pineau, J., Roy, N., Thrun, S., and Verma, V. Experiences with a Mobile Robotic Guide for the Elderly, National Conf. on Artificial Intelligence, pp. 587-592, 2002.

[6] Michalowski, M. P., Sabanovic, S., and Simmons, R. A Spatial Model of Engagement for a Social Robot, IEEE International Workshop on Advanced Motion Control, pp. 762-767, 2006.

[7] Satake, S., Kanda, T., Glas, D. F., Imai, M. Ishiguro, H., and Hagita, N. How to Approach Humans? : Strategies for Social Robots to Initiate
Interaction, ACM/IEEE Int. Conf. on Human-Robot Interaction, pp. 109-116, 2009.

[8] Yamazaki, K., Kawashima, M., Kuno, Y., Akiya, N., Burdelski, M., Yamazaki, A., and Kuzuoka, H. Prior-to-Request and Request Behaviors within Elderly Day Care: Implications for Developing Service Robots for Use in Multiparty Settings, European Conference on Computer Supported Cooperative Work, pp. 61-78, 2007.

[9] Kanda, T., Glas, D. F., Shiomi, M., and Hagita, N. Abstracting People's Trajectories for Social Robots to Proactively Approach Customers, IEEE Transactions on Robotics, vol. 25, pp. 1382-1396, 2009.

[10] Goffman, E. Behavior in Public Places: Notes on the Social Organization of Gatherings, The Free Press, 1963.

[11] Kendon, A. Features of the Structural Analysis of Human Communicational Behavior, in Aspects of Nonverbal Communication, W. v. R. Engel ed., vol., 1980.

[12] Nakauchi, Y. and Simmons, R. A Social Robot That Stands in Line, Autonomous Robots, vol. 12, pp. 313-324, 2002.

[13] Dautenhahn, K., Walters, M. L., Woods, S., Koay, K. L., Nehaniv, C. L., Sisbot, E. A., Alami, R., and Siméon, T. How May I Serve You? A Robot Companion Approaching a Seated Person in a Helping Context, ACM/IEEE Int. Conf. on Human-Robot Interaction, pp. 172-179, 2006.

[14] Kuzuoka, H., Suzuki, Y., Yamashita, J., and Yamazaki, K. Reconfiguring Spatial Formation Arrangement by Robot Body Orientation, ACM/IEEE Int. Conf. on Human-Robot Interaction, pp. 285-292, 2010.

[15] Sisbot, E. A., Marin-Urias, L. F., Alami, R. and Simeon, T. A Human Aware Mobile Robot Motion Planner, IEEE Transactions on Robotics, vol. 23, pp. 874-883, 2007.

[16] Pacchierotti, E., Christensen, H. I., and Jensfelt, P. Embodied Social Interaction for Service Robots in Hallway Environments, Field and Service Robotics, vol. 25, pp. 293-304, 2006.

[17] Henry, P. Vollmer, C., Ferris, B., and Fox, D. Learning to Navigate through Crowded Environments, IEEE Int. Conf. on Robotics and Automation, 2010.

[18] Bennewitz, M., Burgard, W., Cielniak, G., and Thrun, S. Learning Motion Patterns of People for Compliant Robot Motion, International Journal of Robotics Research, vol. 24, pp. 31-48, 2005.

[19] Pandey, A. K. and Alami, R. A Social Robot Which Guides a Person While Supporting the Person's Activity, Congrès des doctorants EDSYS 2009, 2009.

[20] Gockley, R., Forlizzi, J., and Simmons, R. Natural Person-Following Behavior for Social Robots, ACM/IEEE Int. Conf. on Human-Robot Interaction, pp. 17-24, 2007.

[21] Bergström, N., Kanda, T. Miyashita, T. Ishiguro, H. and Hagita, N. Modeling of Natural Human-Robot Encounters, IEEE/RSJ Int. Conf. on Intelligent Robots and Systems, pp. 2623-2629, 2008.

[22] Glas, D. F., Miyashita, T., Ishiguro, H., and Hagita, N. Laser-Based Tracking of Human Position and Orientation Using Parametric Shape Modeling, Advanced Robotics, Vol. 23, No. 4, pp. 405-428, 2009.

[23] Mutlu, B., Forlizzi, J., and Hodgins, J. A Storytelling Robot: Modeling and Evaluation of Human-Like Gaze Behavior, IEEE-RAS Int. Conf. on Humanoid Robots, pp. 518-523, 2006.

[24] Mutlu, B., Shiwa, T., Kanda, T., Ishiguro, H. and Hagita, N. Footing in Human-Robot Conversations: How Robots Might Shape Participant Roles Using Gaze Cues, ACM/IEEE Int. Conf. on Human-Robot Interaction, pp. 61-68, 2009.

[25] Sidner, C. L., Kidd, C. D., Lee, C., and Lesh, N. Where to Look: A Study of Human-Robot Engagement, International Conference on Intelligent User Interfaces, pp. 78-84, 2004.

[26] Guo, Y., Parker, L., and Madhavan. R. Towards collaborative robots for infrastructure security applications. In Proceedings of the 2004 International Symposium on Collaborative Technologies and Systems, pages 235-240, 2004. 